\title{
Synchronization of distributed robotics control system
}

\author{
Boguslaw Twarog ${ }^{1, *}$ and Ewa Zeslawska ${ }^{2}$ \\ ${ }^{1}$ University of Rzeszow, Faculty of Mathematics and Natural Sciences, Rejtana Str. 16C, 35-959 \\ Rzeszow, Poland \\ ${ }^{2}$ University of Information Technology and Management, Sucharskiego Str.2, 35-225 Rzeszow, \\ Poland
}

\begin{abstract}
The paper presents the novel project that has been proposed and realized together with the implementation of the process of heating thermosetting components based on advanced industrial equipment and software tools. In the fundamental task of optimization, the phenomenon of process synchronization based on a centralized algorithm adapted to the specific requirements of production was taken into account. The proposed research station was optimized and located in the laboratory of the Interdisciplinary Computer Modelling of the University of Rzeszow, that specializes in raising the efficiency of industrial processes
\end{abstract}

\section{Introduction}

Present industrial development requires more and better solutions in the field of production control and management. Optimizing these processes in terms of quality, speed, reliability, and safety enables to achieve higher quality and profitability. As a result of specialist consultations that have been carried out with employees from different companies, such as Prat\&Whitney (aerospace components manufacturer) or Borg Warner (automotive manufacturer), it seems that appropriate design and realization of production control system is not an easy task and is often problematic. A frequent issue is the communication problem between different systems of techno-logical lines, especially the synchronization of processes between them, for multidimensional production in particular. Thus, in order to avoid delays and losses in production various security forms are used, such as redundant systems or manual control over particular parts of lines.

Multiple analyses and comparisons of practical solutions that are presented in specialist literature or scientific papers allow to implement suggested optimum and efficient models of final projects. Authors very often recommend or orient on the use of artificial intelligence methods in various industrial processes that pose a significant part of complex technological solutions. The methods of communication and trans-mission between processes and related algorithms suggest that the solution proposed in the present paper is a current approach to the problem $[1,2,3,4]$.

A frequently controlled object requires a comprehensive solution which, next to dispersed control, will ensure full visualization of work and archiving of process data, and will deliver report tools. In general, the synchronization is the overall mechanism with the 
aim of assuring the access of processes to the shared system resources. The problem with synchronization is also present in designing of production process control system. As far as programming of systems with multiple production processes is concerned, the issue of mutual exclusion with the use of critical section is crucial. When one process enters the critical section, it is sure that another process cannot enter it at the same time. Access to the critical section should be regular for all waiting processes and should support mutual exclusion and support limited waiting (no "hunger" effect) [5, 6]. A coordinated mechanism, that is centralized algorithm, was used in order to provide processes with access to a mutual resource. Among available pro-cesses, one is selected to be a coordinator, to which other processes send queue re-quests. If the entry to the critical section is free, the coordinator sends it information. When it is received, the process can enter the critical section. When it leaves the critical section, it must inform the coordinator, and then the coordinator can give access to another process (Fig.1).

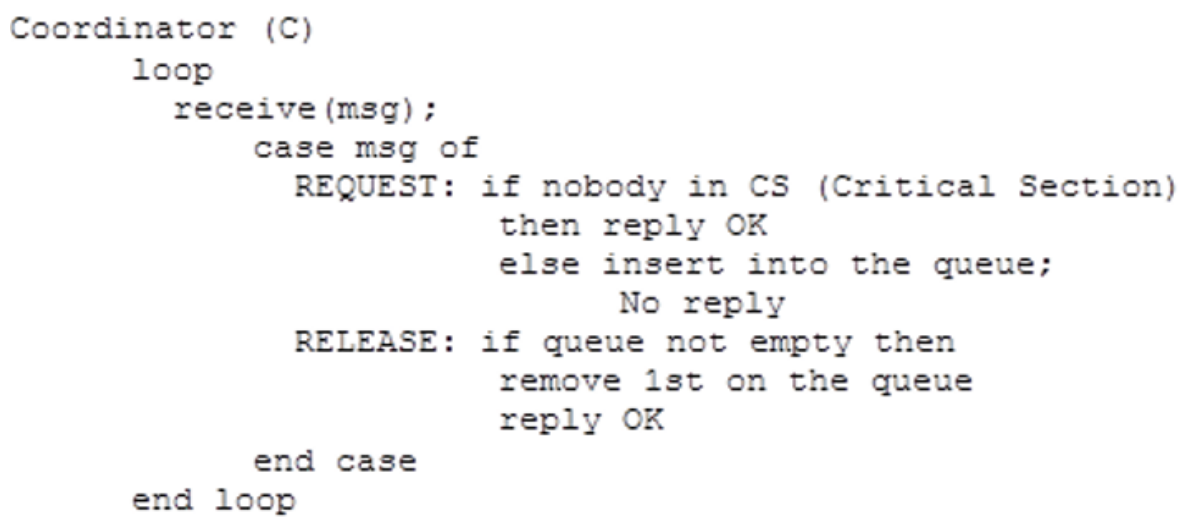

Fig. 1. Centralized algorithm of mutual exclusion (pseudocode)

As a result of conducted analyses, the algorithms were created, programs and visualisations were prepared and proper hardware architecture was selected. During the design and implementation, great attention was paid to the way of synchronization of the programmed production processes. Taking into account the properties of the synchronization algorithm, after sufficient adaptation the concept was used in the designed control system $[7,8$

\section{The analysis and project of the heating process of thermosetting components}

In order to realize and implement the project with success, the analysis was carried out and after that exact assumptions connected with the technological process were determined:

-heating process of thermosetting components will be a major production task;

-components will be delivered from two lines to two accumulators;

-vacuum furnace will be responsible for the heating process;

-components will be delivered to the furnace with the use of two robots, one per each accumulator;

-thermoset components will be put back on the collection belt by robots;

-synchronization of activities between system nodes will be required.

A driver is the most important element in the production process, as it coordinates the work of devices (robots and furnace) and will be responsible for distribution of tasks to particular devices. Appropriately designed LD management program must reflect the 
requirements set by the assumptions of centralized algorithm. According to this, the waiting processes will be technological lines number 1 and 2 that deliver components which are collected in accumulators. The main driver will allow them access to the furnace when needed. Robots will be activated interchangeably. When one of the accumulators is empty, only the robot which has components for transportation will operate. A general scheme of the operation of main driver which controls the work of lines is shown in the algorithm scheme, Fig. 2.



Fig. 2. An essential part of the main algorithm of heating process control. 
All the processes were divided into cycles. Robot work cycle starts when a robot receives a signal that a components can be transported from accumulator to furnace. Next, the robot informs that the task is completed and send an informative signal that it is in a safe position, outside the furnace. When the heating cycle is over, the robot receives a signal that the components can be taken out of the furnace. The whole robot work cycle ends when the robot gives information about the completion of the removal task [9-14].

\section{Equations and mathematics}

The evolution of programmable systems that control processes allows the implementation of advanced control algorithms. Their proper deployment and optimization significantly influences the quality of production management. Configuration of proper communication between system elements also plays an important role. In the project of synchronization of the two technological line processes, the following control system devices were used [15 18]:

-Astraada PLC (HERCC972) drivers: programmable driver dedicated for small and medium industrial facilities. Equipped with discrete 8/4 inputs/outputs, analogue $8 / 4$ inputs/outputs, $128 \mathrm{~KB}$ central processing unit dedicated for control program and the processor realizing the program with the speed of $0.016 \mathrm{~ms} / \mathrm{kB}$. The program for a driver can be implemented in a few standard languages (LD, ST, IL, FBD, SFC).

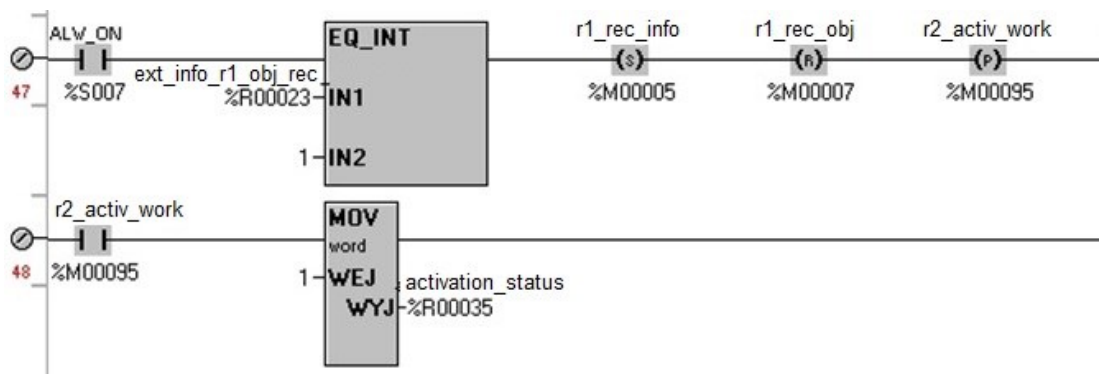

Fig. 3. Part of the code responsible for signal reception informing about the object delivery and about setting proper record marked as the activation status.

The part of control logic, presented above, poses an appropriately adapted implementation of tasks synchronization with the use of selected centralized algorithm. The parts that are in charge of activation and work switch between robots are responsible for giving components from accumulators (process queue) the access to the furnace (critical section). Removing an object from the furnace by a robot is equivalent to the completion of a particular part of the heating process of the component (critical section is over, accumulator is empty) [19 - 21].

-Astraada HMI (AS43TFT0425) touch panels: 4.3" programmable panel for visualization and control over programmed processes. Equipped, among others, with Ethernet communication ports, RS-232, USB, MicroSD. 


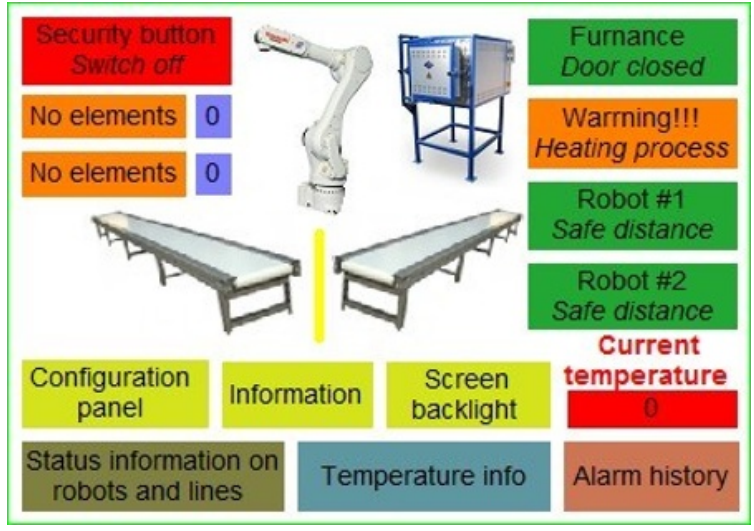

Fig. 4. Main synaptic screen for the lines control driver.

The visualisation or driver support was prepared (fig.4) which enables to collect da-ta from the whole production line. The data were grouped and divided for various screen.

-Manageable JetNet 4510 Ethernet switches: work well in applications in which high reliability and connection efficiency are required. Equipped with 7 ports 10/100TX and 3 combo RJ-45/SFP ports.

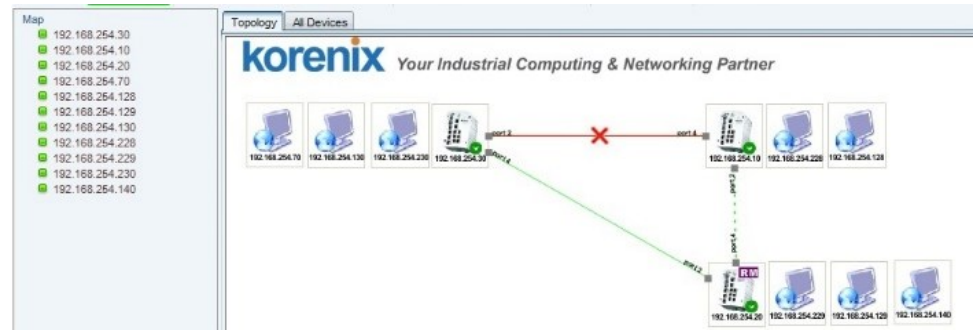

Fig. 5. Checking the operation of Rapid Super Ring function after disconnection one of the transmission medium.

Super Ring is an important, implemented function, used for constructing a ring-structured redundant system. In the testing process, disconnecting one of the transmission medium proved that the communication between processes moved to the back-up circuit and data transfer was not interrupted Fig.5 [22].

-Kawasaki RS003N robots: six-axis device controlled with the controller E70/E7 series. This device is used where high capacity is not required (up to $3 \mathrm{~kg}$ ), but high speed is required $(6000 \mathrm{~mm} / \mathrm{s})$, as well as precision and repeatability of operations $( \pm 0,05 \mathrm{~mm})$. In addition, it is equipped with a pneumatic grab and a SensoPart set for industrial image processing.

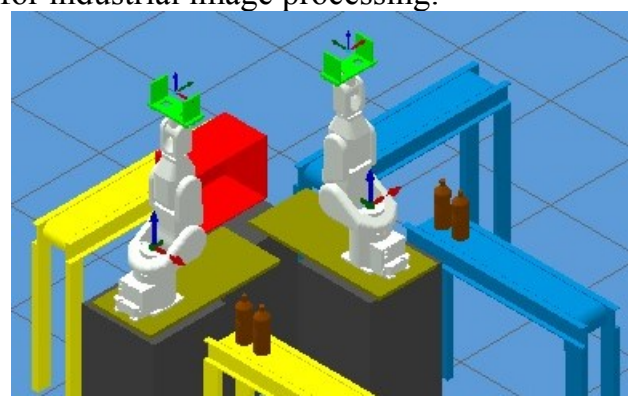

Fig. 6. A simulation of the part of line realized in the K-ROSET program 


\section{Remarks and conclusion}

In the presented solution, a real production process that realizes dedicated heating of thermosetting components was designed and implemented. The modelling and configuration process of dispersed industrial automation novel systems based on PLC drivers and robots with multiple degrees of freedom was presented. The methods and original algorithms of synchronization of various processes were also discussed in order to achieve an optimum control over production lines. The project and its practical implementation using available components and devices underwent a verification process and advanced testing plans. All assumptions that were set have been realized, and the project component parts in verification stages have undergone optimization and adjustment to the project requirements. In a complex phenomenon of dispersed control, known from computer systems centralized algorithm was applied, that is used for process synchronization. Its implementation in ladder language (LD) was not easy and it had to be adapted to the designed situation. Centralization of processes is not the right solution, as the failure of the main driver may stop the work of production line. The appropriate solution would be a possible redundancy of this system that is going to be realized in the nearest future investigations.

\section{References}

1. E. Dudek-Dyduch, Modeling manufacturing processes with disturbances - a new method based on algebraic-logical meta-models, 14th International Conference, ICAISC 2015, (2015)

2. E. Jezierski, Dynamics of robots, (WNT, in Polish, Warszawa), (2006)

3. B. Twarog, Z. Gomolka, E. Zeslawska, Time Analysis of Data Exchange in Distributed Control Systems Based on Wireless Network Model, Analysis and Simulation of Electrical and Computer Systems. Lecture Notes in Electrical Engineering, vol 452. pp 333-342, (2018)

4. B. Twaróg, Z. Gomółka ,E. Żesławska, P. Krutys, The system that supervises and controls the course of the technological process, (in Polish, Education-TechniqueInformatics, 4 (2), 424-430, University of Rzeszow), (2013)

5. B. Wittenmark, J. Nilsson, M. Torngren, Timing problems in real-time control systems, Proceedings of American Control Conference, (1995)

6. A. Noriaki, S. and other, Distrbiuted Component Middleware for RT (Robot Technology), (IEEE/RSJ International Conference on Intelligent Robots and Systems, 10.1109/ACC.1995.531240), (2005)

7. F. Maturana, W. Shen, D.H. Norrie. MetaMorph, An Adaptive Agent-Based, Architecture for Intelligent Manufacturing, (International J. Production Research, Vol.37, No.10, pp. 2159-2174), (1999)

8. W. Nawrocki, Distributed measurement systems, (WKŁ, Warsaw), (2016)

9. I. Hallmann, B. Siemiątkowska, Artificial landmark navigation system, (9th, Int. Symposium Intelligent Robotic Systems. pp. 219-228), (2001)

10. J. Zając, Distributed control of automated production systems, (Monograph 288, Mechanic Series, in Polish, Krakow University of Technology Publisher), (2003)

11. Z. Gomolka, E. Dudek-Dyduch, Y.P. Kondratenko, From Homogeneous Network to Neural Nets with Fractional Derivative Mechanism, Artificial Intelligence and Soft Computing. vol 10245. Springer, Cham, (2017) 
12. G. Dralus, Z. Gomolka, Seasonal models of energy forecasting in photovoltaic systems using artificial neural networks, (International Interdisciplinary PhD Workshop 2017 , Springer, to be published), (2017)

13. Z. Gomolka, Neurons' Transfer Function Modeling with the Use of Fractional Derivative. DepCoS-RELCOMEX 2018. Advances in Intelligent Systems and Computing, vol 761, (2019)

14. E. Dudek-Dyduch, Z. Gomolka, Neural network design without learning, 6th International Conference on Neural Networks and Soft Computing Location: Zakopane, Neural Networks and Soft Computing Book Series: Advances in Soft Computing, pp: 179-187, (2003)

15. M. Pawlak, Z. Żarczyński, The distributed measurement system for the diagnosis of industrial electric drives, (Problem Notebooks - Electric Machines No 1/2013 (98)), (2013)

16. P. Tatjewski, Advanced control of industrial objects: structures and algorithms, (EXIT, Warsaw), (2002)

17. K. Dzierżek, M. Rećko, D.S. Pietrala, Quick prototyping of manipulator control system with PLC controller, (23rd International Conference Engineering Mechanics 2017, pp.294-297), (2017)

18. J. Kofman, X. Wu, T.J. Luu, S. Verma, Teleoperation of a Robot Manipulator Using a Vision-Based Human-Robot Interface, (IEEE Transactions on Industrial Electronics", Vol. 52, Iss. 5, pp.1206-1219), (2005)

19. J. Bartman, Accuracy of reflecting the waveforms of current and voltage through their spectrum determined by the standards regulating measurements, (Revue Roumaine Des Sciences Techniques-Serie Electrotechnique Et Energetique, vol. 61), (2016)

20. J. Bartman, The analysis of output voltage distortion of inverter for frequency lower than the nominal, (Journal of Electrical Engineering, vol 68, No 3, pp.194-199), (2017)

21. T. Binkowski, K. Buczek, D. Sobczynski, Control of BLDC motor working as flying hybrid platform component, (Electrotechnical Review (Przegląd Elektrotechniczny), vol. 87, no 8, pp. 30-33), (2011)

22. V. Cicirello, S. Smith. Ant Colony Control for Autonomous Decentralized Shop Floor, Routing. (Proc. of the 5th Int. Symposium on Autonomous Decentralized Systems., IEEE Computer Society, pp. 383-390), (2001) 